\title{
A Facile One-pot Synthesis of New Pyrazolopyrimidines and Pyrazolo Pyridines Derivatives
}

\author{
Hatem Abdel Moniem Ahmed \\ Department of Forensic Chemistry, \\ College of Forensic Sciences, \\ Naif Arab University for Security Sciences, \\ Riyadh, Saudi Arabia. \\ Hatemahmed29@yahoo.com
}

\begin{abstract}
A Simple Facile One-pot reaction, novel and efficient rout for the synthesis of substituted pyrazolo [3, 4- $d$ ] pyrimidines, and pyrazolo [3, 4-b] pyridines, results from reaction of substituted-5-amino-4-cyanopyrazoles with malononitrile and diethylmalonate respectively. The structures of the products and conceivable mechanisms are discussed. The antibacterial activity of some new synthesized compounds was evaluated and seemed to be significant.
\end{abstract}

\section{Indexing terms/Keywords}

substituted-5-amino-4-cyanopyrazoles; pyrazolo [3, 4- $d$ ] pyrimidines; pyrazolo [3, 4-b] pyridines; diethylmalonate; malononitrile and antibacterial activity.

\section{Academic Discipline And Sub-Disciplines}

Organic Chemistry

\section{SUBJECT CLASSIFICATION}

Organic Chemistry

\section{TYPE (METHOD/APPROACH)}

Some new nitrogen bridge-head pyrido[1,2-b][1,2,4]triazepines incorporating 6-methylchromone moiety have been synthesized from the reaction of 1,6-diamino-4-(6-methyl-4-oxo-4H-chromen-3-yl)-2-oxo-1,2-dihydropyridine-3,5dicarbonitrile (4) with some $\alpha, \gamma$-bifunctional electrophiles including 2-cyano-3,3-bis(methylthio)acrylonitrile, 2-cyano-3,3bis(methylthio)prop-2-enamide, 5-chloro-3-methyl-1-phenylpyrazole-4-carboxaldehyde, 2-chloro-3-formylquinoline, $p$ methoxybenzylidene-malononitrile, ethyl 2-cyano-3-(4-methoxyphenyl)prop-2-enoate, chromone-3-carbonitrile. Structures of the newly synthesized products have been deduced upon the help of elemental analysis and spectral data. The synthesized compounds were screened for their antimicrobial activity.

\section{Council for Innovative Research}

Peer Review Research Publishing System

Journal: Journal of Advances in Chemistry

Vol. 8, No. 1

editor@cirworld.com

www.cirjac.com, member.cirworld.com 


\section{INTRODUCTION}

Pyrazolopyrimidines are of considerable chemical and pharmacological importance as purine analogues, it is a heterocyclic chemical compound with the molecular formula $\mathrm{C}_{6} \mathrm{H}_{5} \mathrm{~N}_{3}$. It forms the central core of a variety of more complex chemical compounds including some pharmaceuticals and pesticides ${ }^{(1,2)}$

Pyrazolopyrimidines have antitumor, antileukemic activities. The pyrazole containing compounds have practical applications in the medicinal and agrochemical field and the biological activity ${ }^{(3-5)}$ of pyrazoles and its derivatives is well documented. The pyrazole ring has shown to be the basic moiety for a number of dyes, drugs and anesthetics ${ }^{(6,7)}$. Amino and hydroxyl substituted pyrazoles have been used as choline esterase inhibitors. Pyrazolopyridines and its hydroderivatives are very interesting pyrazole derivatives with wide ranging biological activities ${ }^{9}$. A number of pyrazolo [3, 4-b] pyridines exhibit a wide range of biological activities, including interesting anxiolytic activity (e.g. tracazolate), dopamine D3 receptor antagonist, antiherpetic and antiallergic properties ${ }^{10}$.

The pyrazolo $[3,4-b]$ pyridine system has interesting biological and pharmacological properties ${ }^{11}$, such as $A C T H$ (adrenocorticotropic hormone) - releasing factor (CRF) (corticotropin-releasing factor)) antagonist activity; CRF antagonists are believed to be effective in the treatment of a wide variety of stress-related illnesses, such as depression, Alzheimer's disease, gastrointestinal diseases, anorexia nervosa, hemorrhage stress, drug and alcohol withdrawal symptoms, drug addiction and infertility ${ }^{12}$. For this purpose we started from the key intermediates (1a-c) (substituted-5amino-4-cyanopyrazoles) (Scheme 1) and reacted them with malononitrile and diethylmalonate to obtain pyrazolo [3, 4- $d]$ pyrimidines and pyrazolo[3,4-b]pyridines respectively.

\section{RESULTS AND DISCUSSION}

The aim of this work, The 5-amino-1H-pyrazole-4-carbonitrile (1), were used as starting materials, which contains a cyano group in the ortho position is required for the synthesis of the condensed systems including pyridine and pyrimidine, with malononitrile and diethylmalonate.

In ethanolic sodium ethoxide solution, compounds (1a) and (1b) reacted with malononitrile to afford pale brown powders of $\mathrm{mp} 235-237{ }^{\circ} \mathrm{C}$ for $(\mathbf{2 a})$ and $289-291^{\circ} \mathrm{C}$ for $(\mathbf{2 b})$, respectively. The ${ }^{1} \mathrm{H}$ NMR spectrum of compound (2a) revealed a methylene singlet at (d) $4.17 \mathrm{ppm}$ and pyrazole $\mathrm{H}-3$ as a singlet at $8.35 \mathrm{ppm}$. Two singlet appear, one $\mathrm{NH}_{2}$ group at $8.07-$ $8.10 \mathrm{ppm}$ and other at 11.04 for $\mathrm{NH}$ as expected. Based on these data it seemed that $\mathrm{a}-\mathrm{CH}_{2} \mathrm{CN}$ side chain is present and that the cyclization took. By addition of the $\mathrm{NH}_{2}$ in the pyrazole (1) to the $\mathrm{CN}$ of the malononitrile to form the amidine intermediate followed by an attack of the newly formed amino group to the $\mathrm{CN}$ of (1) to afford the pyrazolopyrimidine (2), as shown in Scheme 1. On the contrary, reaction of compounds (1a, b) with diethylmalonate in ethanolic sodium ethoxide solution gave pyrazolopyrimidines $(\mathbf{3} \mathbf{a}, \mathbf{b})$. The structure of compounds $(\mathbf{3})$ was confirmed by ${ }^{1} \mathrm{HNMR}$ spectroscopic data. The ${ }^{1} \mathrm{HNMR}$ spectrum of compound (3a) revealed the ester group as a triplet for the $\mathrm{CH}_{3}$ protons at $1.37 \mathrm{ppm}$ and a quartet for the $\mathrm{CH}_{2}$ protons at $4.48 \mathrm{ppm}$, pyrazole $\mathrm{H}-3$, one $\mathrm{NH}_{2}$ group, and an $\mathrm{OH}$ signal at $12.42 \mathrm{ppm}$ (Scheme 1) ${ }^{13}$. It has been found that when compound (1a) refluxing in ethanol in the presence of triethylamine afforded a brown crystalline solid of $\mathrm{mp} 247-249^{\circ} \mathrm{C}$. It was expected that this reaction would give the pyrazolopyrimidine (4a). However, the microanalytical data showed that this product has the molecular formula $\mathrm{C}_{8} \mathrm{H}_{8} \mathrm{~N}_{8}$. Furthermore, the IR spectrum displays an absorption at 3472 and $3312 \mathrm{~cm}-1$, corresponding to $\mathrm{NH}_{2}$ stretching and at 3212 for $(\mathrm{NH}) \mathrm{cm}^{-1}$ and no $\mathrm{CN}$ absorption. The ${ }^{1}$ HNMR spectrum reveals two singlets for the amino groups at 5.77 and $5.99 \mathrm{ppm}$ and two singlets for the pyrazole $\mathrm{H}-3$ protons. Structure (4a) was thus suggested for this product. The formation of compound (4a) may be envisaged via initial condensation of the amino group of one molecule of the o-aminonitrile with the cyano group of a second molecule to give an intermediate amidine which then undergoes a second, but intramolecular, amine-nitrile condensation to give the isolated product. A similar result had been established by Taylor and Borror in the formation of (4a) (Scheme 1$)^{14}$. Compounds (1)-c) were refluxed under the same reaction conditions to afford $(\mathbf{4 b}-\mathbf{c})$. Similar cyclizations with other nitriles have been reported ${ }^{15}$. In an attempt to introduce a formyl group at position 3 in pyrazole (1), aminopyrazole (1) was reacted with Vilsmeier reagent $\left(\mathrm{DMF}-\mathrm{POC}_{13}\right)$ at $70^{\circ} \mathrm{C}$ for $3 \mathrm{~h}$. To the product which was obtained structure (6) was proposed based on the NMR data which indicate the presence of $\mathrm{OH}$ group and the pyrazole $\mathrm{H}-3$, the reaction proceeding via the intermediacy of (5) (Scheme 2).

\section{EXPERIMENTAL}

General Procedures. Melting points are uncorrected. Microanalyses were carried out in the Microanalytical Centre, Cairo University, Egypt. IR (KBr) spectra were measured on a Karl Zeiss IMP 16 spectrophotometer. 1H-NMR spectra were measured by using Jeol

Spectrometer EX-270. Double resonance, HMQC and HMBC experiments were carried out for complete assignment of proton and carbon signals in the NMR spectra, whenever possible. Elemental analyses were obtained on a Leco CHNS932 instrument. Compound 9 was prepared by a known method ${ }^{16}$.

\section{General procedure for preparation of $2 a, b$ and $3 a, b$}

Compound 5-amino-4-cyano-1-H-pyrazole $(\mathbf{1 a}, \mathbf{b}),(10 \mathrm{mmol})$ was added to $(10 \mathrm{mmol})$ malononitrile or diethylmalonate, this mixture was added to $10 \mathrm{~mL}$ freshly prepared sodium ethoxide solution prepared by adding $0.5 \mathrm{~g}$ sodium metal into absolute ethanol $(10 \mathrm{~mL})$ and the mixture was refluxed for $5 \mathrm{~h}$, and left to cool overnight. The solid product so formed was collected by filtration, washed with ethanol and crystallized from ethanol, unless otherwise stated ${ }^{17}$. 
Data:

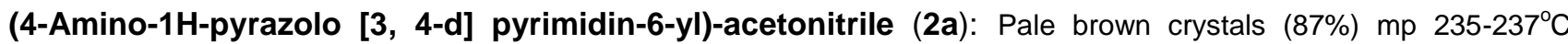
(EtOH). Anal. Calcd for: $\mathrm{C}_{7} \mathrm{H}_{6} \mathrm{~N}_{6}(174.17)$ : C, 48.27; H, 3.71; N, 48.25 \%. Found: C, 48.45; H, 3.65; N, 48.46 \%.IR (KBr) V $=3453$ and $3292\left(\mathrm{NH}_{2}\right), 2215(\mathrm{CN}), 3202(\mathrm{NH}) \mathrm{cm}^{-1} . \delta_{\mathrm{H}}\left(\mathrm{DMSO}_{-} \mathrm{d}_{6}\right): 4.17\left(\mathrm{~s}, 2 \mathrm{H}, \mathrm{CH}_{2}\right), 8.00\left(\mathrm{br} \mathrm{s}, 1 \mathrm{H}, \mathrm{NH}_{2}\right), 8.07(\mathrm{br} \mathrm{s}, 2 \mathrm{H}$, $\left.\mathrm{NH}_{2}\right), 8.10-8.25,8.35$ (s, 1H, H-3) (br s, 1H, NH2), 11.04 (s, 1H, NH); $\delta_{\mathrm{c}}(\mathrm{DMSO}-\mathrm{d} 6): 27.88\left(\mathrm{CH}_{2}\right), 101.13(\mathrm{C}-3 \mathrm{a}), 118.61$ (CN), 135.13 (C-3), 154.78 (C-7a), 159.99 (C-4), 158.55 (C-6).

\section{(4-Amino-1-phenylpyrazolo [3, 4-d] pyrimidin-6-yl)-acetonitrile (2b):}

Brown powder (83\%) mp 289-291 ${ }^{\circ} \mathrm{C}(\mathrm{EtOH})$. Anal. Calcd for: $\mathrm{C} 13 \mathrm{H} 10 \mathrm{~N} 6(250.26)$ : C, 62.39; H, 4.03; N, 33.58. Found C, 62.43; H, 4.18; N, 33.48. IR ( $\mathrm{KBr})$ v: 3462 and 3302, $\left(\mathrm{NH}_{2}\right), 2216(\mathrm{CN}) \mathrm{cm}-1 ; \delta_{\mathrm{H}}\left(\mathrm{DMSO}_{-} \mathrm{d}_{6}\right): 4.14\left(\mathrm{~s}, 2 \mathrm{H}, \mathrm{CH}_{2}\right), 7.23(\mathrm{t}, 1 \mathrm{H}$, $\left.\mathrm{H}-4^{\prime}\right), 7.51$ (d, 2H, H-3, $\left.\mathrm{H}^{\prime} 5^{\prime}\right), 8.12\left(\mathrm{br}, 2 \mathrm{H}, \mathrm{NH}_{2}\right.$ ), 8.27 (d, 2H, H-2, $\left.\mathrm{H}-6^{\prime}\right), 8.35$ (s, 1H, H-3) ); $\delta_{\mathrm{C}}(\mathrm{DMSO}-\mathrm{d} 6): 27.91\left(\mathrm{CH}_{2}\right)$, 100.89 (C-3a), 117.88 (CN), 134.88 (C-3), 154.21 (C-7a), 158.21 (C-4), 159.22 (C-6), 121.46 (C-2', C-6'), 123.22 (C-4'), $128.99\left(\mathrm{C}-3^{\prime}, \mathrm{C}-5^{\prime}\right), 133.22\left(\mathrm{C}-1^{\prime}\right)$.

\section{4-Amino-6-hydroxy-1H-pyrazolo [3, 4-b] pyridine-5-carboxylic acid ethyl ester (3a):}

White powder (87\%) mp 192-194 ${ }^{\circ} \mathrm{C}(\mathrm{EtOH})$; Anal. Calcd for: $\mathrm{C}_{9} \mathrm{H}_{10} \mathrm{~N}_{4} \mathrm{O}_{3}(222.21)$ : C, 48.65; $\mathrm{H}, 4.54 ; \mathrm{N}, 25.21 ; \mathrm{O}, 21.60 \%$. Found: C, 49.19; H, 4.48; N, 25.41\%. IR (KBr) v: $3212(\mathrm{NH}), 3510$ and 3392, $\left(\mathrm{NH}_{2}\right), 3236(\mathrm{br}, \mathrm{OH}), 1661(\mathrm{C}=\mathrm{O}) \mathrm{cm}-1 ; \delta_{\mathrm{H}}$ (DMSO-d $\mathrm{d}_{6}$ ): $1.37\left(\mathrm{t}, 3 \mathrm{H}, \mathrm{CH}_{3}\right), 4.48\left(\mathrm{q}, 2 \mathrm{H}, \mathrm{CH}_{2}\right), 8.16\left(\mathrm{~s}, 2 \mathrm{H}, \mathrm{NH}_{2}\right), 8.54(\mathrm{~s}, 1 \mathrm{H}, \mathrm{H}-3), 12.42(\mathrm{br}, \mathrm{s}, 1 \mathrm{H}, \mathrm{OH}) ; \delta_{\mathrm{c}}(\mathrm{DMSO}$ d6): $14.99\left(\mathrm{CH}_{3}\right), 62.22\left(\mathrm{CH}_{2}\right), 101,29$ (C-3a), 134.99 (C-3), 149.22 (C-7a), $153.22(\mathrm{C}-4), 165.22(\mathrm{C}-6), 171.22(\mathrm{C}=\mathrm{O})$.

\section{4-Amino-6-hydroxy-1-phenyl-1H-pyrazolo [3, 4-b] pyridine-5-carboxylic acid ethyl ester (3b):}

White powder (88\%) mp 304-305 ${ }^{\circ} \mathrm{C}(\mathrm{EtOH})$; Anal. Calcd for: $\mathrm{C}_{15} \mathrm{H}_{14} \mathrm{~N}_{4} \mathrm{O}_{3}$ (298.30): $\mathrm{C}, 60.40 ; \mathrm{H}, 4.73 ; \mathrm{N}, 18.78 ; \mathrm{O}, 16.09$ \%. Found: C, 60.55; H, 4.83; N, $18.68 \%$. IR (KBr) v: $3212(\mathrm{NH}), 3487$ and 3364, $\left(\mathrm{NH}_{2}\right), 3178(\mathrm{br}, \mathrm{OH}), 1677(\mathrm{C}=\mathrm{O}) \mathrm{cm}-1 ;$ $\delta_{\mathrm{H}}\left(\mathrm{DMSO}-\mathrm{d}_{6}\right): 1.33\left(\mathrm{t}, 3 \mathrm{H}, \mathrm{CH}_{3}\right), 4.37\left(\mathrm{q}, 2 \mathrm{H}, \mathrm{CH}_{2}\right), 7.35\left(\mathrm{t}, 1 \mathrm{H}, \mathrm{H}-4^{\prime}\right), 7.51$ (t, 2H, H-3), $8.16\left(\mathrm{~d}, 4 \mathrm{H}, \mathrm{H}-2^{\prime}, \mathrm{H}-6^{\prime}\right), 8.55(\mathrm{~s}$, $1 \mathrm{H}, \mathrm{H}-3), 8.44$ (s, $1 \mathrm{H}, \mathrm{H}-3), 12.85$ (br s, $1 \mathrm{H}, \mathrm{OH}) ; \delta_{\mathrm{c}}$ (DMSO-d6): $14.29\left(\mathrm{CH}_{3}\right), 61.99\left(\mathrm{CH}_{2}\right), 102.10(\mathrm{C}-3 \mathrm{a}), 135.22(\mathrm{C}-3)$,

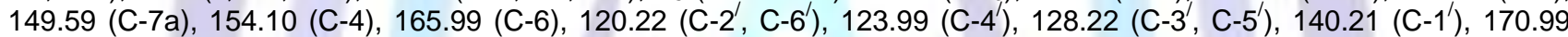
$(\mathrm{C}=\mathrm{O})$.

\section{General procedure for preparation of $4 a-c$ :}

To a solution of 5-amino-4-cyano- pyrazole (1a-c) $(0.1 \mathrm{~mol})$, in ethanol $(10 \mathrm{~mL})$ and triethylamine $(1 \mathrm{~mL})$ was heated under reflux for $7 \mathrm{~h}$ and then concentrated under reduced pressure. The solid product so formed was collected by filtration, washed with ethanol and crystallized from $\mathrm{EtOH}-\mathrm{H}_{2} \mathrm{O}^{17}$.

\section{6-(5-Amino-1H-pyrazol-4-yl)-7H-pyrrolo [2, 3-d] pyrimidin-4-ylamine (4a):}

Brown powder (87\%) mp 247-249 ${ }^{\circ} \mathrm{C}(\mathrm{EtOH})$; Anal. Calcd for: $\mathrm{C}_{8} \mathrm{H}_{8} \mathrm{~N}_{8}(216.21)$ : C, 44.44; $\mathrm{H}, 3.73 ; \mathrm{N}, 51.83 \%$. Found C, 44.34; $\mathrm{H}, 3.79, \mathrm{~N} ; 51.40 \%$. IR (KBr) v: 3472 and 3312, $\left(\mathrm{NH}_{2}\right), 3212(\mathrm{NH}) \mathrm{cm}-1 ; \delta_{\mathrm{H}}\left(\mathrm{DMSO}-\mathrm{d}_{6}\right): 5.77\left(\mathrm{~s}, 2 \mathrm{H}, \mathrm{NH}_{2}-\mathrm{C}-5^{\prime \prime}\right), 5.99(\mathrm{~s}, 2 \mathrm{H}$, $\left.\mathrm{NH}_{2}-\mathrm{C}-4\right), 8.02\left(\mathrm{~s}, 1 \mathrm{H}, \mathrm{H}-3^{\prime /}\right), 8.28(\mathrm{~s}, 1 \mathrm{H}, \mathrm{H}-3), 11.13(\mathrm{~s}, 2 \mathrm{H}, \mathrm{NH}) ; \delta_{\mathrm{C}}(\mathrm{DMSO}-\mathrm{d} 6)$ : 104.21 (C-3a), 141.13 (C-3), $146.22(\mathrm{C}-7 \mathrm{a})$, $155.22\left(\mathrm{C}-5^{\prime \prime}\right), 157.94(\mathrm{C}-4), 160.93(\mathrm{C}-6)$.

\section{6-(5-Amino-1-phenyl-1H-pyrazol-4-yl)-1-phenyl-1H-yrazolo [3, 4-d] pyrimidin-4-ylamine (4b):}

Pale brown powder (77\%) mp 267-269 ${ }^{\circ} \mathrm{C}(\mathrm{EtOH})$; Anal. Calcd for: $\mathrm{C}_{20} \mathrm{H}_{16} \mathrm{~N}_{8}$ (368.40): C, 65.21; $\mathrm{H}, 4.38 ; \mathrm{N}, 30.42 \%$. Found: C, 65.41; H, 4.28; N, 30.62\%. IR (KBr) v: 3472 and 3312, $\left(\mathrm{NH}_{2}\right) \mathrm{cm}-1 ; \delta_{\mathrm{H}}(\mathrm{CDCl}): 6.57\left(\mathrm{~s}, 2 \mathrm{H}, \mathrm{NH}_{2}-\mathrm{C}-5^{\prime \prime}\right), 6.01$ (s,2H, NH $\left.\mathrm{N}_{2}-\mathrm{C}-4\right)$, 7.55-7.62 (m, 2H, Ar-H), 7.88 (appt, 4H, Ar-H), 7.80-7.87 (m, 2H,Ar-H), 8.62 (s, 1H, H-3”), 8.71 (d, $2 \mathrm{H}$, Ar-H), 8.23 (s, $1 \mathrm{H}, \mathrm{H}-3)$;

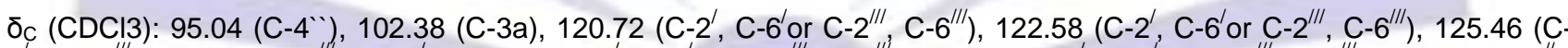
$4^{\prime}$ or C-4 $\left.{ }^{\prime \prime \prime}\right), 126.69\left(\mathrm{C}-4^{\prime \prime \prime}\right.$ or C-4'), $128.66\left(\mathrm{C}-3^{\prime}, \mathrm{C}-5^{\prime}\right.$ or C-3 $\left.3^{\prime \prime \prime}, \mathrm{C}-5^{\prime \prime \prime}\right), 127.64\left(\mathrm{C}-3^{\prime}, \mathrm{C}-5^{\prime}\right.$ or C-3 $\left.3^{\prime \prime \prime}, \mathrm{C}-5^{\prime \prime \prime}\right), 131.33\left(\mathrm{C}-3^{\prime \prime}\right)$, $137.25\left(\mathrm{C}-1^{\prime}\right.$ or $\left.\mathrm{C}-1^{\prime \prime \prime}\right), 138.12\left(\mathrm{C}-1^{\prime \prime \prime}\right.$ or C-1'), 140.03 (C-3), 146.89 (C-7a), 155.19 (C-5 $\left.{ }^{\prime \prime}\right), 157.14$ (C-4), 159.42 (C-6).

\section{6-(5-Amino-1-methyl-1H-pyrazol-4-yl)-1-methyl-1H-yrazolo [3, 4-d] pyrimidin-4-ylamine (4c):}

Brown powder (82\%) mp 265-267 ${ }^{\circ} \mathrm{C}(\mathrm{EtOH})$; Anal. Calcd for: $\mathrm{C}_{10} \mathrm{H}_{12} \mathrm{~N}_{8}(244.26)$ : C, 49.17; H, 4.95; N, $45.87 \%$. Found: C, 49.19; H, 5.25; N, $45.56 \%$. IR ( KBr) v: 3387 and 3329, $\left(\mathrm{NH}_{2}\right) \mathrm{cm}-1 ; \delta_{\mathrm{H}}\left(\mathrm{DMSO}-\mathrm{d}_{6}\right): 2.45\left(3 \mathrm{H}, \mathrm{s}, \mathrm{H}_{3}\right), 2.46\left(3 \mathrm{H}, \mathrm{s}, \mathrm{H}_{3}\right), 6.74$ (s, 2H, NH ${ }_{2}-\mathrm{C}-5$ "), 7.45 (br, 2H, NH $\left.\mathrm{NH}_{2}-\mathrm{C}-4\right), 7.88$ (s, 1H, H-3), 8.36 (s, 1H, H-3"); $\delta_{\mathrm{C}}$ (DMSO-d6): 20.99 (CH3), $20.61(\mathrm{CH} 3)$ $98.99\left(\mathrm{C}-4^{\prime \prime}\right), 102.99(\mathrm{C}-3 \mathrm{a}), 121.11\left(\mathrm{C}-2^{\prime}, \mathrm{C}^{\prime} 6^{\prime}\right), 123.22\left(\mathrm{C}-2^{\prime}, \mathrm{C}^{\prime} 6^{\prime}\right), 129.33\left(\mathrm{C}-3^{\prime}, \mathrm{C}-5^{\prime}\right), 130.22\left(\mathrm{C}-3^{\prime}, \mathrm{C}^{\prime} 5^{\prime}\right), 135.22\left(\mathrm{C}-4^{\prime}\right)$, $135.99\left(\mathrm{C}-4^{\prime}\right), 136.22\left(\mathrm{C}-1^{\prime}\right), 135.22\left(\mathrm{C}-1^{\prime}\right), 139.99(\mathrm{C}-3), 146.99$ (C-7a), 157.55 (C-4), 160.91 (C-6).

\section{General procedure for preparation of $(6 a, b)$ :}

$10 \mathrm{mmol}$ of $(\mathbf{1 a}, \mathbf{b})$ was added to phosphoryl chloride $(1.915 \mathrm{~g}, 12.5 \mathrm{mmol})$ in anhydrous DMF $(2.5 \mathrm{~mL})$ was heated under stirring at $70^{\circ} \mathrm{C}$ for $3 \mathrm{~h}$. Then, the reaction mixture was poured onto ice and treated with aqueous ammonia ( $\left.\mathrm{pH} 8\right)$. A white solid separated and it was filtered off, washed with water, dried and recrystallized from an appropriate solvent to afford the products in $60-82 \%$ yields.

\section{H-Pyrazolo [3, 4-d] pyrimidin-4-ol (6a):}

White powder (75\%) mp 301-302 ${ }^{\circ} \mathrm{C}(\mathrm{EtOH})$; Anal. Calcd for: $\mathrm{C}_{5} \mathrm{H}_{4} \mathrm{~N}_{4} \mathrm{O}(136.11)$ : C, 44.12; $\mathrm{H}, 2.96 ; \mathrm{N}, 41.16 ; \mathrm{O}, 11.75 \%$. Found: C, 44.29; H, 2.75; N, 41.36; O, $11.85 \%$. IR (KBr) v: $3212(\mathrm{NH}), 3237(\mathrm{br}, \mathrm{OH}) \mathrm{cm}-1$; $\delta_{\mathrm{H}}\left(\mathrm{DMSO}-\mathrm{d}_{6}\right): 8.19(\mathrm{~s}, 1 \mathrm{H}, \mathrm{H}-$ 
6), 8.45 (s, 1H, H-3), 12.35 (br, 1H, OH); $\delta_{\mathrm{c}}$ (DMSO-d6):106.98 (C-3a), 136.22 (C-3), 149.22 (C-6), 151.23 (C-7a), 158.11 (C-4).

\section{1-Phenyl-1H-pyrazolo [3, 4-d] pyrimidin-4-ol (6b):}

White powder (87\%) mp 311-312 ${ }^{\circ} \mathrm{C}(\mathrm{EtOH})$; Anal. Calcd for: $\mathrm{C}_{11} \mathrm{H}_{8} \mathrm{~N}_{4} \mathrm{O}(212.21)$ : C, 62.26; $\mathrm{H}, 3.80 ; \mathrm{N}, 26.40 ; \mathrm{O}, 7.54 \%$. Found: C, 62.39; H, 3.65; N, 26.56; O, $7.75 \%$. IR (KBr) v: $3247(\mathrm{br}, \mathrm{OH}) \mathrm{cm}-1 ; \delta_{\mathrm{H}}\left(\mathrm{DMSO}-\mathrm{d}_{6}\right): 7.41\left(\mathrm{t}, 1 \mathrm{H}, \mathrm{H}-4^{\prime}\right), 7.58(\mathrm{t}$, $2 \mathrm{H}, \mathrm{H}-3^{\prime}$ and $\left.\mathrm{H}^{-} 5^{\prime}\right), 8.13\left(\mathrm{~d}, 2 \mathrm{H}, \mathrm{H}-2^{\prime}, \mathrm{H}^{\prime} 6^{\prime}\right), 8.21$ (s, $\left.1 \mathrm{H}, \mathrm{H}-6\right), 8.39$ (s, 1H, H-3), $12.45(\mathrm{br}, 1 \mathrm{H}, \mathrm{OH})$.

\section{Antibacterial activity}

The moiety in pyrazole known for their popular pharmacological activities ${ }^{(19,20)}$, moiety in pyrazole, both in the form of a substituent or as a fused component, changes its properties and converts it into an altogether new and important heterocyclic derivative. Pyrimidines have attracted particular interest over the last few decades due to the use of such a ring system as the core nucleus in various drugs ${ }^{19}$. They are well considering the importance of pyrazolopyrimidine derivatives for their biological activity, it was thought worthwhile to test most of our prepared compounds (2 a,b - 3a,b, $\mathbf{4 a}$ c, 5a-c, 6a,c) for their antibacterial activity against some bacteria namely Escherichia coli, Pseudomonas aeruginosa, Staphylococcus aureus and Enteroccus faecalis. The minimum inhibitory concentrations (MIC) were ascertained by the broth dilution method (microdilution using 96 -well microplates) ${ }^{20}$. The results presented in table 1 showed that $3 \mathbf{b}$, $4 \mathbf{c}$ were the most active towards Pseudomonas aeruginosa. We also noticed that adding a $\mathrm{CH}_{2}$ in the fragment decreases this activity. Compound $\mathbf{4 c}$ was the most active against E. coli, Acinetobacter, Pseudomonas Aeruginosa, and Staphlococcus Aureus. The remaining compounds were found to have a slight or moderate activity against the tested organisms.

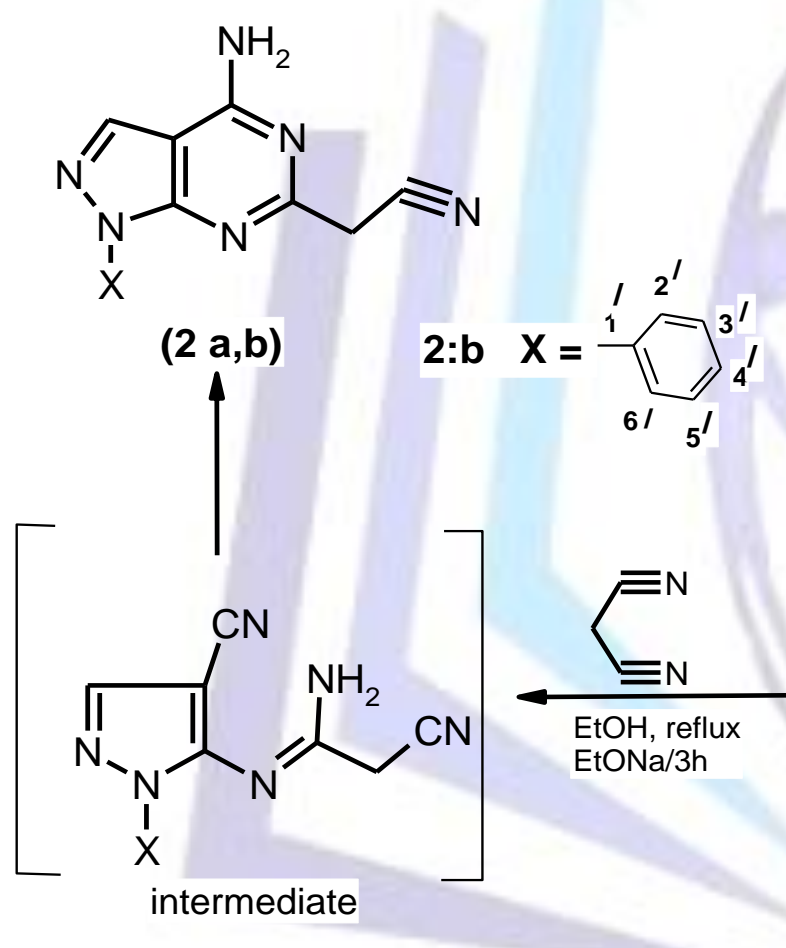

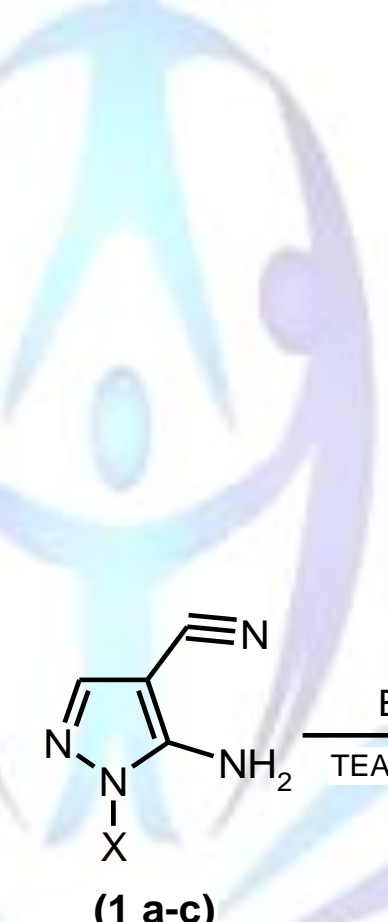

$(1 \mathrm{a}-\mathrm{c})$<smiles>CCOC(=O)COc1ccc(OCC)cc1</smiles><smiles>[X]n1ncc2c(N)c(C(=O)OCC)c(O)nc21</smiles>

$(3 a, b)$

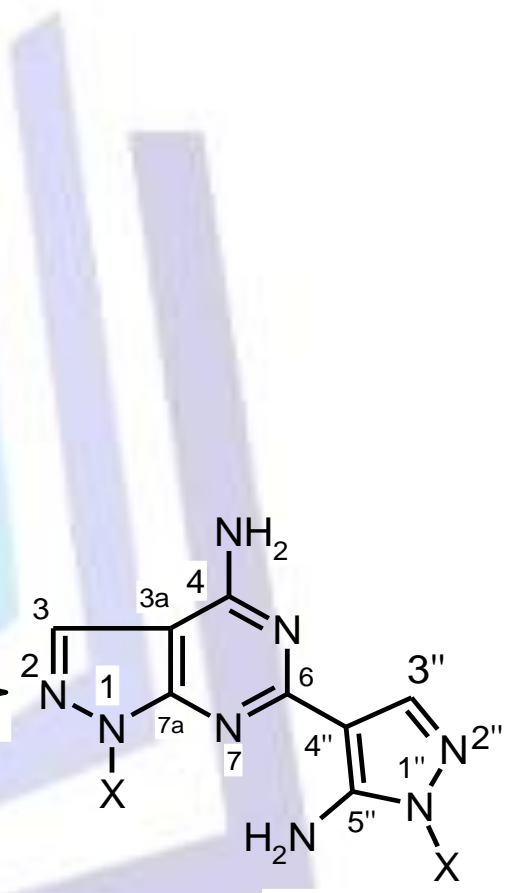

(4 a-c)

$\mathrm{b}: \mathrm{X}=\mathrm{Ph}$

c: $\mathrm{X}=\mathrm{CH}_{3}$

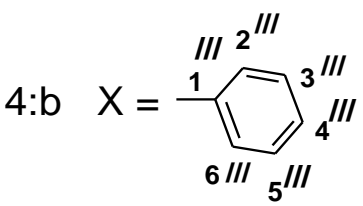


<smiles>[X]n1ncc(C#N)c1N</smiles>

(1 a-c)
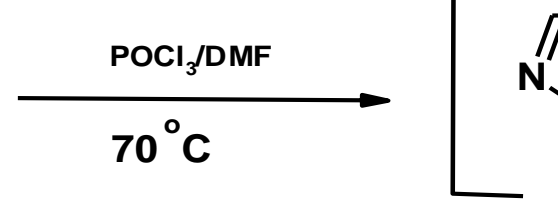

(5)

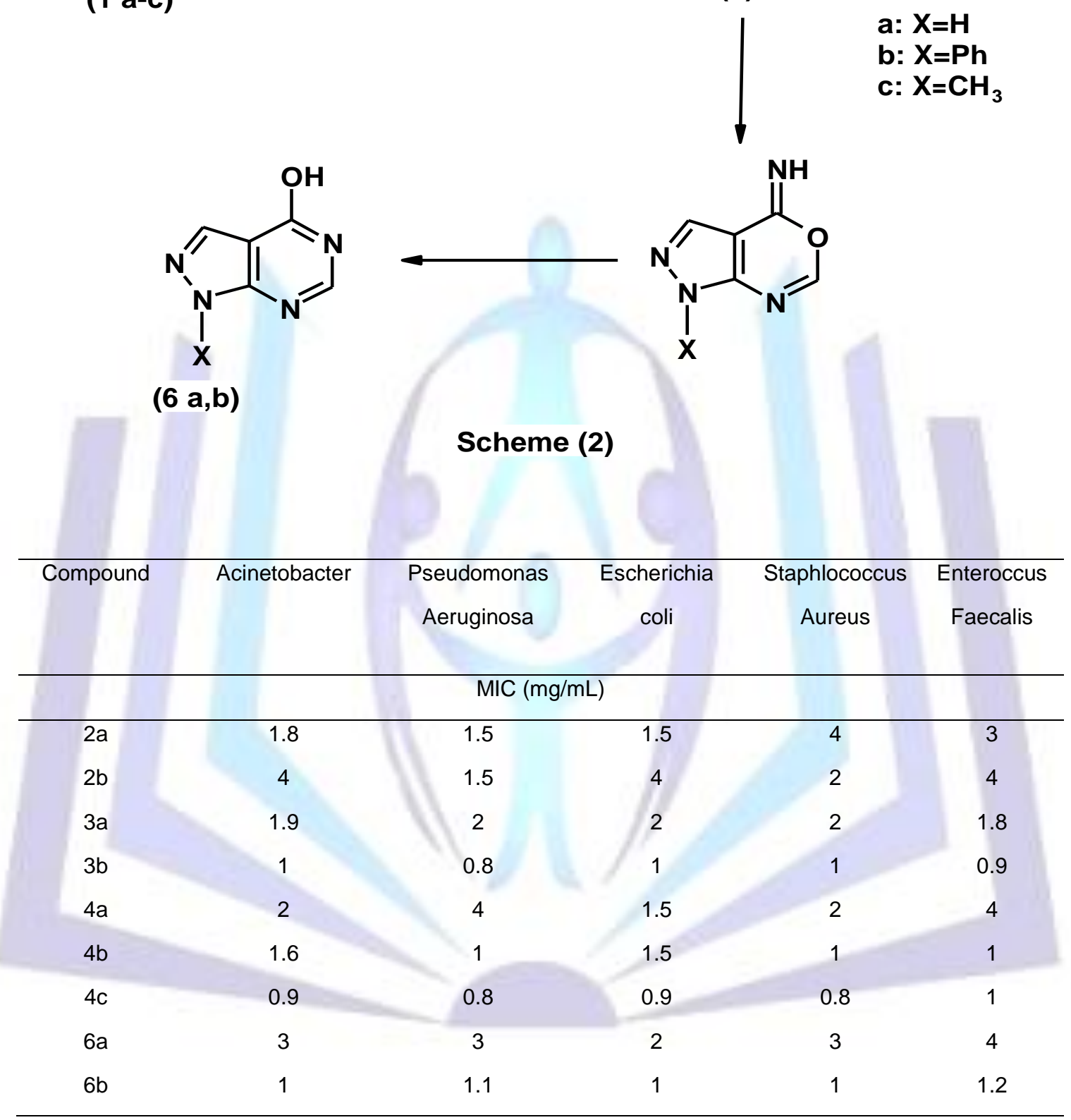

Table 1 Antibacterial activity of some synthesized compounds

\section{REFERENCES}

1. Bendich, A.; Russell, P. J. Jr.; Fox, J. J. J. Am. Chem. Soc. 1954, 76, 6073.

2. Kabayasahi, S. J. Pharm. Bull. 1973, 21, 941.

3. Novinson, T.; Dimmit, R. M. K.; Simon, I.N.; Robins, R. K.; Brien, D. E. O. J. Med. Chem. 1974, 17, 645.

4. Krikpatrick, W. E.; Okabe, T.; Hillyard, I. W.; Robins, R. K.; Dren, A. T.; Novinson, T. J. Med. Chem. 1977, $20,386$. 
5. Elagamy, A. A.; El-Taweel, F. M. A.; Amer, F. A.; Zoorob, H. H. Arch. Pharm. (Weinhei) 1987, 320, 246.

6. (a) Kost, A. N.; Grandberg, I. I. In Advances in Heterocyclic Chemistry Katritzky, A.R. Ed. Academic Press: New York, 1996, p 347. (b) Nihset, H. B. J. Chem. Soc. 1938, 1568.

7. (a) Raiziss, G. W.; Clemence, L. W.; Friefelder, M. J. Am. Chem. Soc. 1941, 63, 2739. (b) Guarneri, M. Bull. Chim. Far. 1960, 99, 259. compounds - Pyrazolones

8. (a) Olah, G. A.; lyer, P. S.; Prakash, S. Synthesis, 1982, 523. (b) Weisberger, A. "The Chemistry of Heterocyclic Pyrazolidones and derivatives", New York, 1964; Vol. 20, pp 1 - 539.

9. (a) Hardy, C. R. Adv. Heterocycl. Chem. 1984, 36, 343. (b) Yu, G.; Mason, H.; Wu, X.; Wang, J.; Chong, S.; Dorough, G.; Henwood, A.; Pongrac, R.; Seliger, L.; He, B.; Normandin, D.; Adam, L.; Krupinski, J.; Macor, J. J. Med. Chem. 2001, 44, 1025. (c) El-Ahl, A. A. S.; Metwally, M. A.; Amer, F. A. Boll. Chim. Farm. 1995, 7, 369.

10. (a) Bettinetti, L.; Schlotter, K.; Hübner, H.; Gmeiner, P. J. Med. Chem. 2002, 45, 4594. (b) Löber, S.; Hübner, H.; Utz, W.; Gmeiner, P. J. Med. Chem. 2001, 44, 2691. (c) Kuroda, S.; Akahane, A.; Itani, H.; Nishimura, S.; Durkin, K.; Tenda, Y.; Sakane, K. Bioorg. Med. Chem. 2000, 8, 55. (d) Johns, B. A.; Gudmundsson,; Turner, K. S. E. M.; Allen, S. H.; Samano, V. A.; Ray, J. A.; Freeman, G. A.; Boyd, F. L.; Sexton, C. J.; Selleseth, D. W.; Creech, K. L.; Moniri, K. R. Bioorg. Med. Chem. 2005, 13, 2397. (e) Gudmundsson, K. S.; Johns, B. A.; Wang, Z.; Turner, E. M.; Allen, S. H.; Freeman, G. A.; Jr, F. L. B.; Sexton, C. J.; Selleseth, D. W.; Monirib, K. R.; Creech, K. L. Bioorg. Med. Chem. 2005, 13,5346 .

11. (a) Hardy, C. R. Adv. Heterocycl Chem. 1984, 36, 343. (b) Orth, R. E. J. Pharm. Sci. 1968, 57, 537. (c) Elnagdi, M. H.; Elmoghayar, M. R. H.; Elgmeie, G. E. H. Adv. Heterocycl. Chem. 1987, 41, 319. (d) Elnagdi, M. H.; Elmoghayar, M. R. H.; Sadek, K. U. Adv. Heterocycl. Chem. 1990, 48, 223.

12. Chen, Y. L. International Patent WO9534563 A1, Chem. Abstr. 1995, 124, 232447.

13. Veronese, A. C.; Callegari, R.; Salah, S. A. Tin (VI) chloride-promoted reaction of b-dicarbonyl compounds with nitrile. Synthesis of aminopyridines and aminoquinolines. Tetrahedron. Lett. 1990, 31, 3485-3488.

14. Taylor, E. D.; Borror, A. L. The reaction of Nitriles with o-Aminonitriles: Aconvenient synthesis of fused 4Aminopyrimidine. J. Org. Chem. 1961, 26, 4967-4974.

15. Smyrl, N. R.; Smithwick, R. W. Hydroxide-catalyzed synthesis of heterocyclic aromatic amine derivative from nitriles. J. Heterocyclic Chem. 1982, 19, 493-496.

16. Cheng, C. C.; Robins, R. K. Potential purine antagonists. VI. Synthesis of 1-Alkyland 1-Aryl-4-aubatituted pyrazolo[3,4d]pyrimidines. J. Org. Chem. 1956; 21; 1240-1256.

17. Abdellatif, M. Salaheldin; Ana, M. F. Oliveira-Campos and Lígia M. Rodrigues. Heterocyclic synthesis with nitriles: Synthesis of pyrazolopyrimidine and pyrazolopyridine derivatives An International Journal for Rapid Communication of Synthetic Organic Chemistry, 2009, 1186-1195.

18. El-Sayed, T. Eur. J. Med. Chem. 2009, 44, 4385-4392.

19. Ghorab, M. M.; Ragab, F. A. Alqasoumi, S. I.; Alafeefy, A. M.; Aboulmagd, S. A. 490 Eur. J. Med. Chem. 2010, 45, 171-178.

20. Abunada, N. M.; Hassaneen, H. M.; Kandile, N. G.; Miqdad, O. A. Molecules. 487, 2008, 13, 1501-1517. 


\section{Author' biography with Photo}

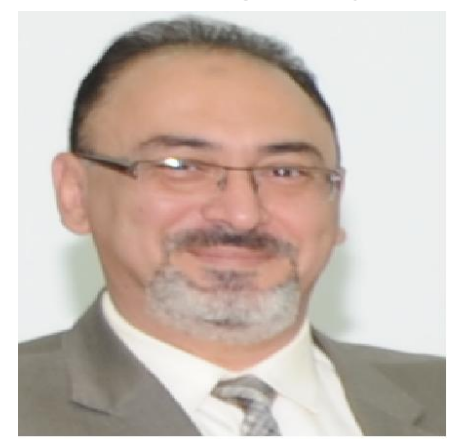

Dr. Hatem Abdel Moniem Ahmed, Egyptian, (born 1968), Current position: Head of Crime Scene Department, Prof. Assistant Forensic Toxicology, Department of Forensic Chemistry, College of Forensic Sciences, Naif Arab University for Security Sciences (NAUSS)- Riyadh- KSA, 1990 B.Sc., 1990, Chemistry, Faculty of Science, Assiut University, Sohag branch, Egypt, M.Sc., 2007 Chemistry Dep., Faculty of Science, Minia University, Egypt, PhD, 2011, Chemistry Dep.,Faculty of Science, Minia University, Egypt. He published more than 26 Papers in Specialized Journals and international conferences. Dr. hatem, member in numerous scientific societies, and has won numerous Awards and Prizes in the internal and external conferences.

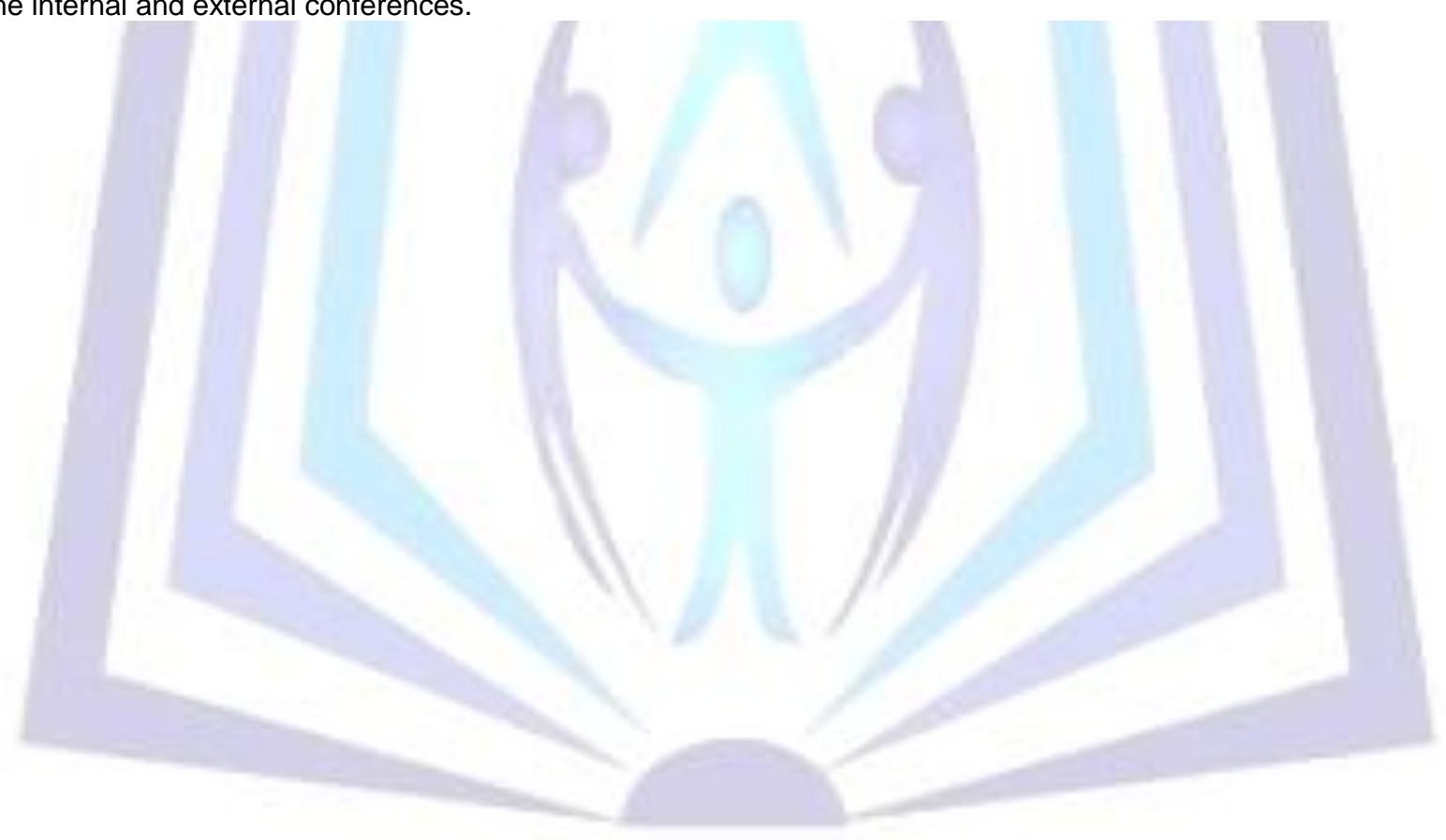

